\title{
Exosuits give a leg up
}

Science 365, 668-672 (2019)

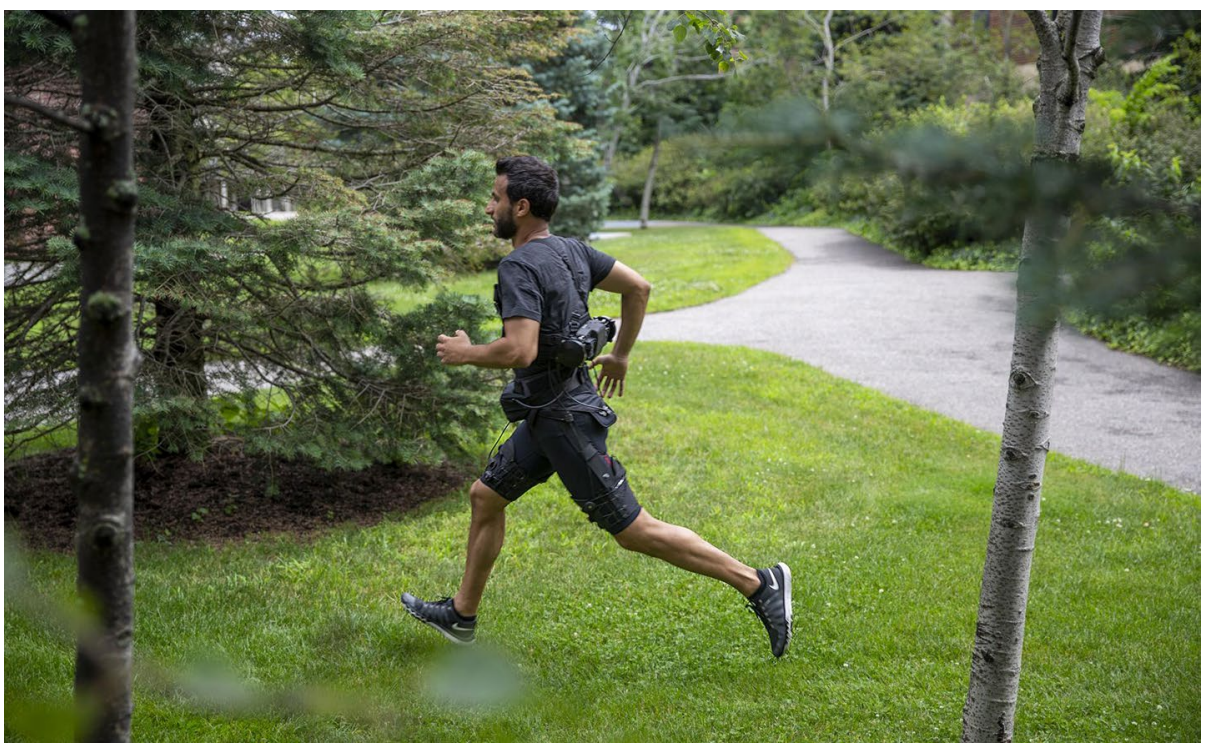

Credit: Wyss Institute at Harvard University

The biomechanical performance of human limbs can be enhanced with wearable electronic systems. Designing these systems to suit a broad range of tasks with varying requirements is a key challenge. Running and walking, for example, have dissimilar biomechanics. For walking, the largest internal joint moments occur at the ankle, whereas for running the largest moments occur at the knee and ankle. Philippe Malcolm, Conor Walsh and colleagues at Harvard University, Chung-Ang University and the University of Nebraska Omaha have now developed a soft, wearable exosuit that can detect whether the user is running or walking and switch the type of assistance to suit the gait accordingly.
The system first collects data from inertial measurement units to determine the user's gait. Then, depending on whether the user is running or walking, the exosuit provides the appropriate biomechanical assistance. The exosuit provides a reduction in metabolic rate to the user of $9.3 \%$ when walking (at a speed of $1.5 \mathrm{~m} \mathrm{~s}^{-1}$ ) and a reduction of $4 \%$ when running (at a speed of $2.5 \mathrm{~m} \mathrm{~s}^{-1}$ ). Though the reduction in metabolic rate appears modest, the researchers suggest that the savings can lead to meaningful improvements in athletic performance.

\section{Michael Lee}

Published online: 17 September 2019

https://doi.org/10.1038/s41928-019-0311-5 